\title{
A Concept for Robust, High Density Terminal Air Traffic Operations
}

\author{
Douglas R. Isaacson", John E. Robinson III." and Harry Swenson ${ }^{\dagger}$ \\ NASA Ames Research Center, Moffett Field, CA 94035 USA \\ Dallas G. Denery \\ University of California Santa Cruz, Moffett Field, CA 94035 USA
}

\begin{abstract}
This paper describes a concept for future high-density, terminal air traffic operations that has been developed by interpreting the Joint Planning and Development Office's vision for the Next Generation (NextGen) Air Transportation System and coupling it with emergent NASA and other technologies and procedures during the NextGen timeframe. The concept described in this paper includes five core capabilities: 1) Extended Terminal Area Routing, 2) Precision Scheduling Along Routes, 3) Merging and Spacing, 4) Tactical Separation, and 5) Off-Nominal Recovery. Gradual changes are introduced to the National Airspace System (NAS) by phased enhancements to the core capabilities in the form of increased levels of automation and decision support as well as targeted task delegation. NASA will be evaluating these conceptual technological enhancements in a series of human-in-theloop simulations and will accelerate development of the most promising capabilities in cooperation with the FAA through the Efficient Flows Into Congested Airspace Research Transition Team.
\end{abstract}

\section{Introduction}

Sustained air traffic demand growth is a major contributor to economic expansion but has led to congestion and significant delay at the busiest airports in the National Airspace System (NAS). While economic cycles offer brief reprieves from this trend, the Joint Planning and Development Office (JPDO) was created to address the long-term needs of the U.S. airspace system: increased capacity, improved environmental performance, and enhanced security, while maintaining or improving safety. ${ }^{1}$ The JPDO has proposed broad application of emerging technologies in aircraft Communication, Navigation and Surveillance (CNS) to address the predicted capacity shortfall while simultaneously enhancing safety and reducing environmental impact (fuel, noise and emissions). The advent of Global Positioning System (GPS)-based aircraft operations including Required Navigational Performance (RNP) brings a powerful tool to the air traffic management community; precision aircraft operations are envisioned with known intent and high predictability.

Advanced navigational systems and procedures alone will not reach the goals of NextGen. RNP procedures in complex, high-density airspace present significant problems for traditional management of off-nominal events and inherent system uncertainties; path and (to a lesser extent) speed flexibility are virtually lost. Further, busy airports in close proximity to one another place additional constraints on flows to and from those airports, causing tunneling of departures, step-down arrivals and other inefficiencies. Such proximate airport systems (or "metroplexes") will require new procedures and increased coordination to avoid significant environmental impact. What new mechanisms will be needed, and the roles of the air traffic controller and supporting automation systems in the future system are unclear. How traffic managers and controllers utilize automation and decision support to respond to off-nominal events and mitigate inherent uncertainties is also undetermined. While automation of routine and redundant tasks is likely, the importance of human involvement in the management of complex systems is widely

\footnotetext{
* Aerospace Engineer, Aerospace High Density Operations Branch, M/S 210-6, AIAA Senior Member.

${ }^{\dagger}$ Research Scientist, Aerospace High Density Operations Branch, M/S 210-6, AIAA Member.

\$ Senior Scientist, AIAA Fellow.
} 
acknowledged.-5 The future role of the human manager or controller in routine (but safety critical) operations has not been clearly defined, but will be critical to the success of NextGen.

The purpose of NASA's Super Density Operations (SDO) research area is to enable significantly increased and robust throughput at the busiest airports and most congested metroplexes while minimizing environmental impact. Complex airspace such as that within the New York or Southern California Terminal Radar Approach Controls (TRACONs) exhibit a level of complexity and congestion that is not seen en route and requires a new approach to terminal air traffic management. The goal of this paper is to describe a concept that will integrate new CNS technologies while providing mechanisms to manage offnominal events and inherent system uncertainties. The concept provides for a transition from today's operations to a terminal system that eventually relies on automation for a large portion of routine operations (including scheduling, sequencing, spacing and separation) and suggests leveraging the complex problem solving abilities of humans to manage recovery from off-nominal events. Development of mid-term and end-state capabilities will be coordinated with the Federal Aviation Administration (FAA) through a joint Research Transition Team (RTT) focused on efficient flows in congested airspace, ensuring a common understanding of the NextGen SDO concept and the research requirements for realization of the envisioned operational improvements.

\section{Background}

This section provides an overview of prior work in the field of terminal air traffic operations and research. The concept in this paper concerns arrival operations from prior to top-of-descent to joining an Instrument Approach Procedure (IAP) and departure operations along Standard Instrument Departure (SID) procedures. The relevant prior work is presented in four sub-fields: 1) NASA Decision Support Tools (DSTs), 2) Efficient Descent Procedures, 3) Weather Impact Mitigation, and 4) Human and Automation Roles and Responsibilities. These four sub-fields address the essential elements of terminal operations needed to develop the SDO concept. An additional section briefly summarizing critical lessons learned from operational activities is included to highlight the difficulties associated with bringing advanced procedures and decision support to congested terminal airspace.

\section{A. NASA Terminal Automation Decision Support Tools}

A significant body of work precedes the development of the concept proposed in this paper. Early work at NASA focused on developing DSTs to assist the air traffic controller and traffic managers at Air Route Traffic Control Centers (ARTCCs) and TRACON facilities in managing arriving and departing flows of traffic. This set of tools is called the Center/TRACON Automation System (or CTAS). The Traffic Management Advisor (TMA) is deployed at the ARTCCs throughout the NAS. ${ }^{6}$ The TMA assists ARTCC controllers and traffic managers in meeting scheduled times of arrival (STAs) to closely match the desired separations and Airport Arrival Rate, among other constraints. ${ }^{7}$ A number of other decision support tools were developed as part of CTAS, and some were tested operationally. The Descent Advisor (DA) provides controllers with executable descent clearances that efficiently meet STAs. ${ }^{8}$ DA was operationally evaluated and development of decision support based on DA continued with Tailored Arrivals (TA) and the En Route Descent Advisor (EDA). ${ }^{9,10}$ The passive Final Approach Spacing Tool (pFAST) was developed to assist TRACON controllers with directing flows arriving from the ARTCC to the runway by advising efficient runway assignments and arrival sequences. ${ }^{11}$ pFAST was operationally tested, and was adapted for multiple TRACONs, but is no longer in operation. ${ }^{6,12}$ Multi-center TMA (McTMA) is an enhanced form of the TMA that coordinates flows from multiple ARTCCs feeding an airport close to their boundaries (where the basic TMA would not suffice). ${ }^{13,14}$ Multi-center TMA was operationally evaluated and key elements of the McTMA concept are included in the FAA's Time-Based Flow Management plan (TBFM). The Expedite Departure Path (EDP) tool advises TRACON controllers when unrestricted climbs were available for departing aircraft, thus eliminating inefficient tunneling beneath arrival streams when traffic allowed. ${ }^{15}$ EDP was evaluated in human-in-the-loop simulations, but further tool evolution was considered premature given the uncertainty associated with climb predictions at the time of EDP's initial development, and EDP was not tested operationally. While TMA, DA, pFAST, McTMA and EDP provided ancillary environmental benefits, the primary objective of each was to reduce delay or to increase throughput. 


\section{B. Efficient Descent Procedures}

More recent work has focused on developing procedures for the specific purpose of reducing fuel burn, emissions and noise impact. Research at NASA Langley Research Center was aimed at developing new aircraft guidance algorithms for minimizing noise impact, referred to as Low-Noise Guidance (LNG). ${ }^{16}$ Many forms of enhanced descent procedures have been proposed including: Continuous Descent Arrivals (CDAs) and Oceanic Tailored Arrivals (OTAs) and Optimized Profile Descents (OPDs). ${ }^{17-19}$ Each of these rely on the application of precision navigation in the development of the supporting procedures. This reliance on precision procedures differs from the previously developed DSTs, such as pFAST and TMA, which assumed a large degree of controller intervention to provide separation service while optimizing aggregate flow metrics such as average delay. The integration of single aircraft trajectory optimization through precision procedures and traffic separation and optimization for high-density, complex flows has not been directly addressed in the literature. Becher and MacWilliams have proposed a concept for merging aircraft on uncoordinated precision arrival routes that uses capabilities built upon existing terminal automation (i.e., Converging Runway Display Aid and Relative Position Indicator). ${ }^{20,21}$ Ren and Clarke have developed a method that allows for a significant level of trajectory optimization on precision paths with a reduced degree of controller intervention. ${ }^{22}$ Capozzi investigated the use of TRACON airspace in coordinated vs. segregated operations for coupled airport systems; his work suggests the possibility of using a mix of temporal coordination and segregated procedures to mitigate the effects of crossing flows in metroplex airspace. ${ }^{23}$ Neither the DST development nor the trajectory optimization efforts have addressed robustness to weather phenomena or off-nominal events.

\section{Weather Impact Mitigation}

Krozel et al. proposed the application of Snell's law to define paths through terminal airspace when convective weather can be treated as deterministic. ${ }^{24,25}$ They further developed an algorithm to estimate capacity over longer time frames when weather is treated probabilistically, using computational geometry and ensemble weather forecasts to assist in defining routes with a high probability of availability. ${ }^{26-28}$ Rhoda et al. investigated translation of convective weather forecasts to terminal area impacts. ${ }^{29}$ Michalek and Balakrishnan have begun to look at predictability of route availability in TRACON airspace, providing insight into how airspace requirements for RNP procedures might change when convective weather is expected in terminal airspace. ${ }^{30}$

\section{Human and Automation Roles and Responsibilities}

One goal in the development of the SDO concept is to identify the limits of controller separation responsibility with the technologies and tools envisioned in the NextGen timeframe. Because one of the key attributes of NextGen in general, and of SDO specifically, is to enable a significant increase in air traffic operations, steps must be taken to mitigate controller workload in providing separation services. This paper proposes an incremental approach to modifying the controller's responsibilities, in line with the controller's ability to safely provide services at increased traffic levels and with automation assistance. Prevot and Palmer have conducted a series of simulations investigating controller roles and responsibilities in future concepts with increasing levels of technology (e.g., data communication) and automation support (e.g., spacing guidance). ${ }^{31,32}$ NASA Langley researchers have developed a system for managing spacing from the flight deck with speed advisories, and have tested this system operationally. ${ }^{33,34}$ Erzberger has proposed a system known as Automated Airspace Concept to nearly fully automate en route air traffic operations by automating separation service and conflict avoidance, and has demonstrated this concept for en route operations as well as arrival operations with metering constraints. ${ }^{35,36}$ Effective roles for the human controller, traffic manager, pilot, automation systems and decision support will be critical to the mitigation of inherent system uncertainties and the recovery from off-nominal events.

\section{E. Lessons Learned}

This section summarizes critical lessons learned through the development and use of CTAS tools, the development and use of efficient descent procedures, and analysis of current day operations. Four lessons that must be considered in the definition of the SDO concept are: 


\section{Lesson 1: Feasible schedules will remain critical}

Building on the foundational work of Roger Dear, TMA researchers first noted the importance of feasible arrival schedules. ${ }^{7,37,38}$ It was found through simulation that schedules that deviated significantly from a first-come first-served sequence resulted in high controller workload and were often deemed 'unworkable,' or infeasible. The desire to execute precision procedures places greater burden on automation systems in regard to the feasibility of advised actions. Infeasible advisories or sequences would require aborting the precision procedures that form the foundation of NextGen. The ability of an ATC automation system to define solutions and advisories that are not only feasible, but deemed reasonable and appropriate by the humans involved in their execution (i.e., pilots and/or controllers), has proven difficult.

\section{Lesson 2: Efficient procedures can be implemented without new technology}

Efficient descent procedures are beginning to be used during moderately busy arrival periods at some airports (e.g., OPDs at Los Angeles International Airport (LAX) and OTAs at San Francisco International Airport). Figure 1 shows the routes for a number of LAX OPD arrivals; shortly after entering Southern California TRACON airspace, aircraft cross 'tie-points' on the routes. If aircraft on these routes cross their respective tie-points at the same time, this indicates to the controller that they are expected to arrive at the downstream merge (LUVYN) at the same time. Unfortunately, the TMA metering resolution (1 minute) is insufficient for avoiding ties. Increased TMA metering delay resolution (e.g., $1 / 10^{\text {th }}$ minute) and/or scheduling to tie points could eliminate the vast majority of OPD interventions. LAX OPD arrivals are merged with other, conventional step-down arrivals from different directions; these conventional flows retain significant path flexibility at merge onto final approach. By retaining these other flows as conventional (step-down) descents, it is not necessary to replace the TMA scheduler as would likely be needed if all arrivals had the reduced temporal control of precision routes/descents. Similar strides are being made in the departure realm through the deployment of RNAV SIDs that remove procedural restrictions or flow dependencies limiting departure throughput. ${ }^{39}$

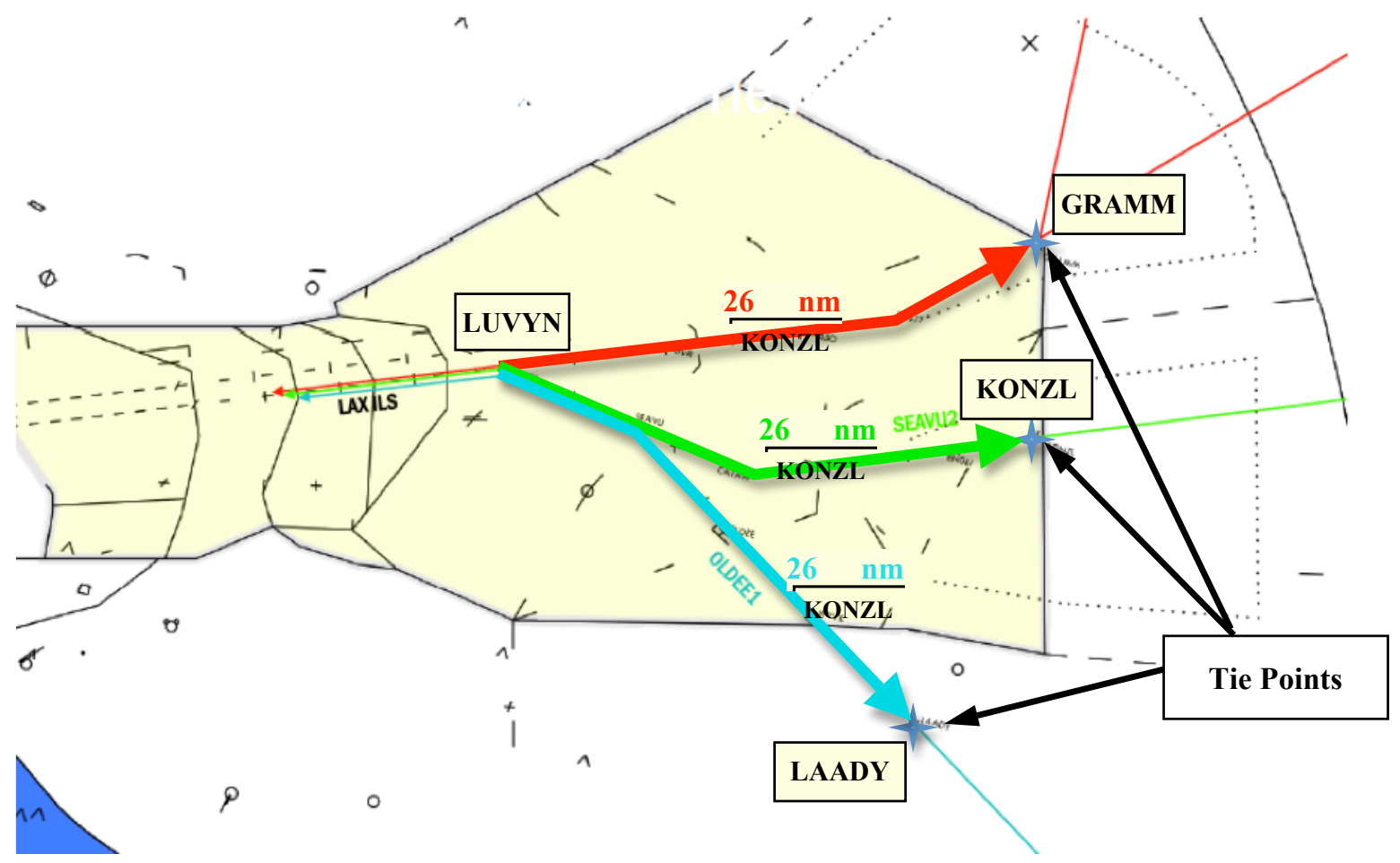

3. Lesson 3: Efficient procedures must not be pursued at the expense of throughput at the busiest airports

Previous studies have suggested that peak runway throughput could be reduced anywhere from $10 \%-50 \%$ if idle descent CDAs are pursued without advanced controller decision support and precision 4D approaches. ${ }^{40,41}$ Shrestra et al. simulated daytime OPD operations for Denver International Airport and 
found only $15 \%$ of potential CDA benefits were realized when optimized descents were limited to an altitude range between 15,000 feet and 25,000 feet to avoid conflicts with other en route and departure flows. ${ }^{42}$ However, Verhoeven showed that the application of airborne spacing technologies with a relaxed descent flight path angle can reduce or eliminate the throughput penalty associated with idle descents, ${ }^{43}$ and Clarke has developed OPD procedures that approximate a CDA and show similar benefit while limiting the number of required controller interventions. ${ }^{22}$ However, predictions of the magnitude of expected benefit from improved descent procedures have varied considerably (up to 3000lb of fuel per flight saved). ${ }^{10}$ Recent work by Robinson and Kamgarpour suggests that when metering delay or aircraft separation requires path-stretching, CDAs exhibit diminishing benefit. ${ }^{44}$ As will be discussed in following sections, this finding has profound implications on the proposed SDO concept that appear counterintuitive without the details included in their study.

\section{Lesson 4: Lessons 2) and 3) are not always at odds}

OPD procedures at LAX have demonstrated that it is sometimes possible to optimize a subset of arriving flows without significantly impacting other flows into a major airport, minimizing the aforementioned throughput penalty. Routes arriving into LAX from the east are highly constrained due to flows to and from nearby airports, mountainous terrain, nearby restricted airspace, and alignment with the primary arrival runways; these arrival routes had very little maneuvering room even prior to OPD implementation. Some level-offs on these flows were procedural and unrelated to delay associated with merging of flows to LAX. New procedures could mitigate such inefficiencies without new technology and with no loss in throughput. However, as discussed by Robinson in Reference 44, when path stretching is required, it is of little consequence if that delay is absorbed at cruise speed prior to top of descent, or at lower speeds in terminal airspace.

\section{SDO Concept}

A fundamental assumption of the NASA Super Density Operations (SDO) concept is its heavy reliance on knowledge of aircraft intent for accurate trajectory and demand predictions. However, inherent uncertainties in NAS operations such as weather forecasts, mechanical malfunctions, medical emergencies, capacity fluctuations, and airspace availability highlight the challenge NextGen faces in meeting future needs with a system based on a highly precise, highly predictable CNS infrastructure. The SDO concept addresses this paradox by combining flexible precision procedures with advanced scheduling logic to mitigate uncertainty (whether it be in a controller-managed or automated separation environment). The SDO concept is presented in a functional decomposition framework and phases-in incremental enhancements to the NAS toward realizing the NextGen vision in terminal airspace. The SDO functional architecture is first presented with a description of each of the five core SDO functional elements: 1) Extended Terminal Area Routing, 2) Precision Scheduling Along Routes, 3) Merging and Spacing, 4) Tactical Separation and 5) Off-Nominal Recovery. A detailed description of the enhanced functional capabilities is then presented in each of three implementation phases: near-term, mid-term and end-state.

\section{A. SDO Functional Architecture}

The SDO concept as addressed in this paper is concerned with the domain of operations from roughly (just prior to) top-of-descent to joining the approach on arrival, and wheels up to top-of-climb on departure. En route operations not subject to SDO scheduling constraints are not considered, nor are surface operations or airport configuration/runway configuration management. Figure 2 depicts the functional architecture of the SDO concept and how the five core functional elements relate to one another (i.e., strategic vs. tactical processes and ground-based vs. airborne execution). These functions exist in today's system in some form or another. The roles that humans and automation play in performing these functions will change substantially as technological advances and procedural changes are implemented gradually over time. A high-level description of these functionalities follows, as well as additional comments regarding the controller interface and functional allocation. 


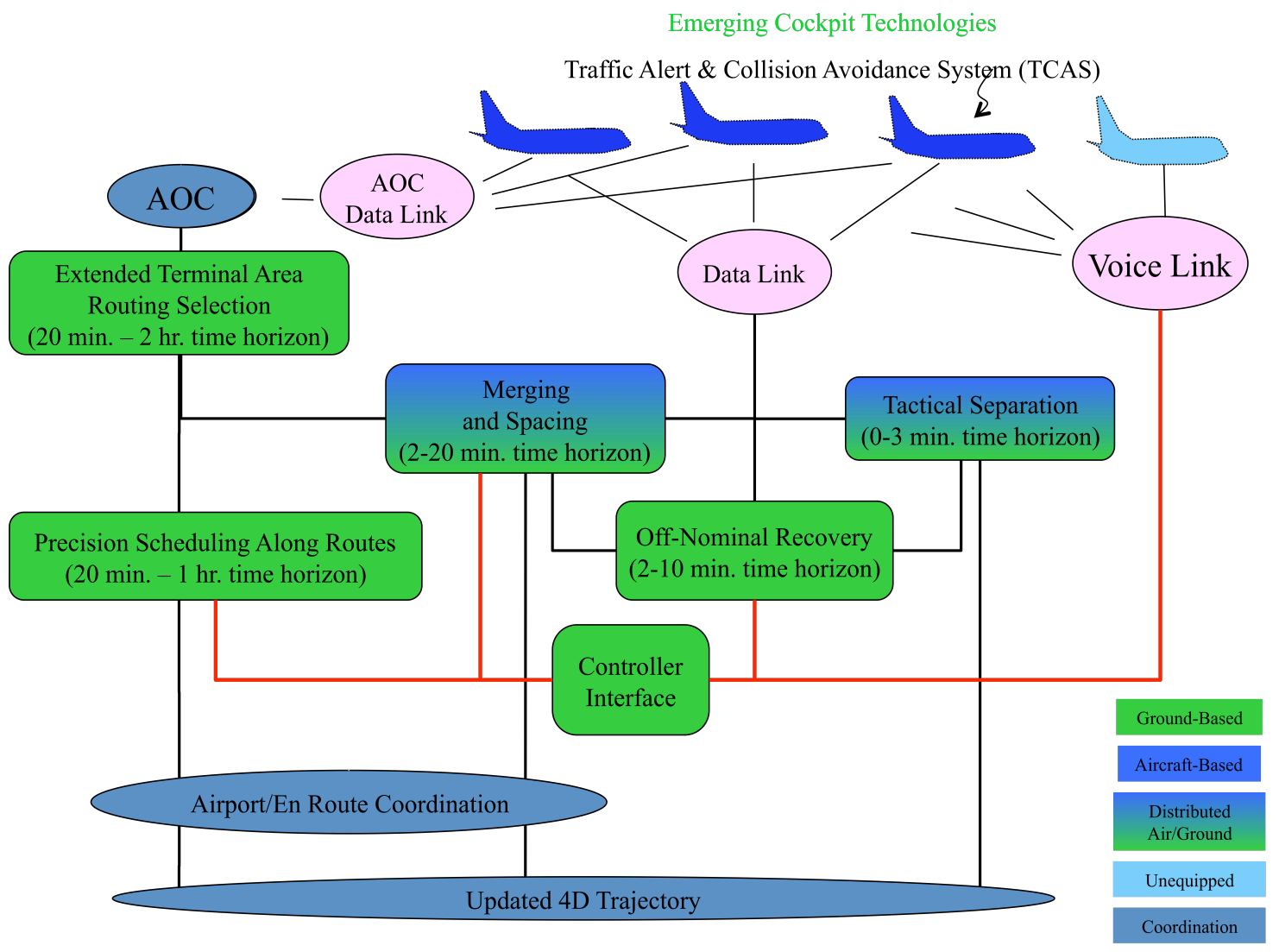

Figure 2: SDO Functional Architecture

\section{Extended Terminal Area Routing}

This function defines available arrival and departure routes that serve the airports in the SDO domain. The route structure is tailored to meet the prescribed airport/airspace configurations while meeting traffic demand requirements, avoiding weather constraints within terminal airspace, and leveraging aircraft capabilities where appropriate (e.g., RNP procedures). The routing function sets the stage for the scheduling function by defining the aircraft routes as a set of clearly defined and predictable procedures.

2. Precision Scheduling Along Routes

The role of the scheduling function for SDO is to organize the flow of aircraft into an efficient sequence in preparation for arrival, and to execute departure operations. The Extended Terminal Area Routing function provides the scheduling function with the framework within which it must determine a scheduling solution. For nominal operations, the scheduling solution is constrained by the defined routing; ad hoc vectoring is not to be relied upon in the determination of the schedule.

\section{Merging and Spacing}

Once the scheduling function has determined an arrival or departure sequence for an aircraft, the Merging and Spacing function provides control instructions to maintain necessary spacing with preceding and successive aircraft along the prescribed route(s). The prescribed spacing is often in excess of required minimum separation to meet traffic flow constraints, in preparation for merging streams of aircraft, or as a consequence of inter-aircraft compression resulting a decelerating flow. The Merging and Spacing function provides spacing between aircraft on the same, or dependent route segments; it is not intended as a primary separation provision function.

\section{Tactical Separation}

Tactical Separation refers to short-term provisions for maintaining required separation between aircraft (whether on common route segments or not), and is a safety function in place as a backup in the uncommon event that higher-level functions result in a predicted loss of separation. Collision avoidance functionality (represented in Figure 2 by TCAS capability), while not addressed in this paper, must be 
considered in the development of the Tactical Separation function to ensure seamless integration from the flight crew perspective.

\section{Off-Nominal Recovery}

Off nominal recovery is an essential safety function of the National Airspace System (NAS). This function is responsible for identifying off-nominal conditions and defining and executing the corrective actions necessary to return to nominal operations. In this context, off-nominal operations are those that do not conform to the expected behavior and recovery is not achievable or desirable with nominal procedures and control mechanisms. Such conditions should be infrequent, but are not to be considered rare events (e.g., missed approach or low fuel state). Off-Nominal Recovery includes a conformance monitoring function to determine when operations fall outside expected (nominal) bounds. A comprehensive approach for SDO conformance monitoring is currently under development and will be detailed in a future publication.

6. Controller Interface and Additional Notes on Functional allocation in Today's NAS

Significant changes in roles and responsibilities proposed for NextGen will necessarily draw clear distinctions between each of the functions that today's controller summarizes as the "safe, orderly and expeditious flow of air traffic ${ }^{45}$ " to ensure roles and responsibilities are clearly defined. Today's system consists of a layered approach that includes Traffic Flow Management, Controller-provided Separation Services, Short-term Conflict Detection/Resolution and Collision Avoidance. The SDO concept will build on this approach to further clarify roles and assign distinct responsibilities to automation, flight crews and air traffic controllers. As depicted by shading in Figure 2, the Merging and Spacing function as well as the Tactical Separation function could be ground-based or flight-deck based; the extent of task delegation to the flight crew and the balance of automation and controller responsibility will be determined through simulation and by the pace of technology adoption in the aircraft fleet. As such, an effective controller interface is critical if the controller is to manage aircraft of mixed equipage in complex terminal airspace. The controller interface will change significantly over the NextGen timeframe to meet the needs of the controller's role and responsibilities.

\section{B. Phased Implementation Strategy and Concept Capability Summary}

The SDO functional architecture is designed to apply equally to today's operations as to fully realized NextGen operations. The core functional components will be gradually replaced with more automated and/or delegated capabilities where appropriate and beneficial. Gradual implementation is intended to provide safe fallback as new capabilities are introduced, while enabling significantly higher capacity over the long-term. It is important to note, however, that as significant demand increases over the long-term, it will not be possible to return to today's capabilities for extended periods of time (recovery transients may still be possible). The remainder of this paper is dedicated to the phased implementation of the five aforementioned functional elements. At each phase, the system is robust and is progressively enhanced for performance gain.

Table 1 provides a summary of the assumptions and functional capabilities in each implementation phase. In general terms, near-term SDO is characterized by compatibility with existing aircraft capabilities and controller practice. All aircraft operating nominally in SDO airspace will be RNAV capable and will communicate with air traffic control via voice. Mid-term SDO is characterized by expanded use of RNAV/RNP Standard Instrument Departure (SID) and Standard Terminal Arrival Route (STAR) procedures in congested airspace. As such, all aircraft will be RNAV capable and most will be RNP capable. Most aircraft will be equipped with minimal (e.g., FANS-1A) data communication, and a limited number of aircraft will be equipped for flight-deck managed spacing. End-State SDO is characterized by a highly capable fleet of aircraft. All aircraft operating in SDO airspace will be equipped for RNP operations and have advanced data communication capabilities. Routine communication with air traffic control will be via data communication and aircraft will navigate precise RNP routes with prescribed altitude and time constraints. 
Table 1: SDO Concept Capability Summary

\begin{tabular}{|c|c|c|c|}
\hline & Near-Term & Mid-Term & Far-Term \\
\hline Assumptions & $\begin{array}{l}\text { - Compatible with existing } \\
\text { fleet equipage } \\
\text { - Mixture of RNAV OPD and } \\
\text { step-down arrivals } \\
\text { - Departure ops rely } \\
\text { primarily on RNAV-based } \\
\text { procedures } \\
\text { - Current controller and pilot } \\
\text { procedures, technologies, } \\
\text { capabilities employed } \\
\text { - RNP procedures are } \\
\text { limited to acute problems of } \\
\text { terrain, procedural } \\
\text { separation and noise } \\
\text { abatement. }\end{array}$ & $\begin{array}{l}\text { - Mixed equipage with Increasing numbers } \\
\text { of aircraft equipped for RNP, data } \\
\text { communication } \\
\text { - RNAV/ RNP SIDs and STARs with } \\
\text { altitude and speed restrictions } \\
\text { - Aircraft controlled to meet STAs at } \\
\text { runway threshold and key merge points } \\
\text { - Controller responsible for aircraft } \\
\text { separation } \\
\text { - Limited number of aircraft capable of } \\
\text { Flight Deck Managed Spacing at } \\
\text { controller's discretion } \\
\text { - Communication via voice, with limited } \\
\text { data }\end{array}$ & $\begin{array}{l}\text { - 3D and/or 4D RNP routes } \\
\text { within TRACON } \\
\text { - Aircraft controlled to meet } \\
\text { STAs at runway, key merge } \\
\text { points, and where inter-flow } \\
\text { coordination is required } \\
\text { - Generation, transmission, } \\
\text { and execution of clearances } \\
\text { for routine ops largely } \\
\text { automated (pilot acceptance } \\
\text { required) } \\
\text { - Most aircraft capable of Flight } \\
\text { Deck Managed Spacing } \\
\text { - Routine communication via } \\
\text { data, voice as backup }\end{array}$ \\
\hline $\begin{array}{l}\text { Extended Terminal } \\
\text { Area Routing Function }\end{array}$ & $\begin{array}{l}\text { - Expanded use of RNAV } \\
\text { OPDs } \\
\text { - Adoption of RNAV SIDs } \\
\text { - Targeted redesign of } \\
\text { grossly inefficient } \\
\text { procedures }\end{array}$ & $\begin{array}{l}\text { - Predefined set of RNAV/RNP } \\
\text { procedures } \\
\text { - Predominantly RNAV OPD arrivals with } \\
\text { limited step-down descents } \\
\text { - Automation selects assigned terminal } \\
\text { procedure from set } \\
\text { - Controller reviews and issues arrival and } \\
\text { departure clearances via voice or data }\end{array}$ & $\begin{array}{l}\text { - Dynamically defined 4D RNP } \\
\text { procedures automatically } \\
\text { cleared via data } \\
\text { communication } \\
\text { - All arrivals conduct efficient } \\
\text { descents (OPD or Tailored } \\
\text { Arrival) } \\
\text { - RNP departures with vertical } \\
\text { restrictions } \\
\text { - Coordination of } \\
\text { arrival/departure and } \\
\text { Metroplex flows }\end{array}$ \\
\hline $\begin{array}{l}\text { Precision Scheduling } \\
\text { Along Routes }\end{array}$ & $\begin{array}{l}\text { - Expanded use of TMA } \\
\text { - Modification of TMA for } \\
\text { RNAV OPDs and RNP } \\
\text { procedures } \\
\text { - Timeline-based decision } \\
\text { support for pairing very } \\
\text { closely-spaced approach } \\
\text { operations }\end{array}$ & $\begin{array}{l}\text { - TMA extended to include merge point } \\
\text { scheduling } \\
\text { - Time advance to close gaps in arrival } \\
\text { streams } \\
\text { - Partial slot recovery to mitigate arrival } \\
\text { variance } \\
\text { - Constrained position shifting from first- } \\
\text { come-first-served }\end{array}$ & $\begin{array}{l}\text { - Automatic transmission of } \\
\text { STAs } \\
\text { - Automatic transmission of } \\
\text { pairing assignment for very } \\
\text { closely-spaced approaches } \\
\text { - Automatic rescheduling as } \\
\text { needed }\end{array}$ \\
\hline Merging and Spacing & $\begin{array}{l}\text { - Largely unchanged } \\
\text { - Controllers use situational } \\
\text { display aids to space aircraft } \\
\text { with speed instead of } \\
\text { vectors }\end{array}$ & $\begin{array}{l}\text { - Decision support for controller-managed } \\
\text { spacing } \\
\text { - Delegated spacing at controller's } \\
\text { discretion }\end{array}$ & $\begin{array}{l}\text { - Ground-based spacing } \\
\text { instruction via data } \\
\text { communication } \\
\text { - Automatic transmission of } \\
\text { delegated spacing assignment }\end{array}$ \\
\hline Tactical Separation & $\begin{array}{l}\text { - Automation alerts } \\
\text { controller of pending loss of } \\
\text { separation } \\
\text { - Controller resolves } \\
\text { conflicts }\end{array}$ & $\begin{array}{l}\text { - Automation alerts of pending loss of } \\
\text { separation and provides resolution options } \\
\text { - Controller selects or defines own } \\
\text { resolution and provides clearance to } \\
\text { aircraft via voice }\end{array}$ & $\begin{array}{l}\text { - Automation predicts imminent } \\
\text { loss of separation } \\
\text { - Automatic generation and } \\
\text { transmission of resolution } \\
\text { clearance } \\
\text { - Controller issues automation- } \\
\text { provided resolution via voice } \\
\text { as data communication back- } \\
\text { up }\end{array}$ \\
\hline Off-Nominal Recovery & - No changes expected & $\begin{array}{l}\text { - Largely manual process } \\
\text { - Traffic Management Unit (TMU) } \\
\text { rescheduling options } \\
\text { - Controller trial planning for reinsertion } \\
\text { trajectories } \\
\text { - Spatial or temporal buffers in place to } \\
\text { mitigate off-nominal events }\end{array}$ & $\begin{array}{l}\text { - Controller remains central } \\
\text { - Decision support for rapid } \\
\text { constraint identification } \\
\text { - Optimal Path Maps available } \\
\text { as catalog of contingency } \\
\text { routes available at any time }\end{array}$ \\
\hline
\end{tabular}




\section{Near-Term SDO Capabilities}

\section{Assumptions for the Near-Term}

Operations in the near-term will need to be compatible with existing fleet equipage. As such, there are few assumptions on required capabilities of aircraft operating in the terminal environment. The following assumptions are made regarding near-term SDO:

- Arrival operations will include a mixture of: 1) RNAV-based OPD STAR procedures from (at a minimum) prior to top of descent all the way to the runway, 2) standard operations relying on nominal interior routes, step-down descents and radar vectoring, and 3) a limited number of tailored arrival procedures for suitably equipped aircraft.

- Departure operations will rely primarily on RNAV-based procedures.

- Current controller and pilot procedures, technologies and capabilities will be employed.

- Limited RNP procedures will be developed to address acute problems associated with flow segregation, terrain avoidance, and noise abatement.

\section{Extended Terminal Area Routing}

Extended Terminal Area Routing in the near term will be characterized by expanded use of RNAV OPDs and widespread adoption of RNAV SIDs. Due to the limited number of aircraft that are equipped for RNP, such procedures will continue to be implemented only where a specific need requires high-precision lateral path definition. For reasons discussed in the following paragraphs, standard, step-down descents will likely remain predominant for most arrival flows, with OPDs being utilized for select flights and flows that provide the most benefit. The identification of routes blocked by weather, encroachment of Special Use Airspace (SUA) or Military Operations Area (MOA) airspace, or violation of TFM constraints will remain a manual process. Routing modifications will be managed as they are today, with controllers issuing clearances via voice transmission.

Improvements to operational efficiency in Extended Terminal Area Routing can be realized through more efficient or predictable procedures using existing technologies. Continued development of RNAV departure and arrival procedures will provide efficiency and capacity improvements by removing procedural restrictions that would otherwise be required.

\section{Precision Scheduling Along Routes}

Enhancements to Precision Scheduling Along Routes will include: 1) expanded use of TMA, 2) modifications to TMA for OPDs, RNAV, and RNP procedures, and 3) assistance with identifying aircraft suitable for Very Closely Spaced Parallel Approach (VCSPA) operations. Expanded controller use of TMA to meter arriving aircraft according to the TMA-scheduled crossing time will increase controller familiarity and proficiency with TBFM techniques that will form the foundation for future scheduling enhancements.

Enhancements to TMA will include modifications to the terminal delay model to accurately reflect the reduced TRACON flight times for OPDs, and might include enhancements to benefit the conduct of OPD arrivals such as increased metering precision or tie-point timelines. TMA enhancements might also include basic assistance to controllers for managing VCSPA operations such as the Simultaneous Offset Instrument Approach (SOIA). While automated algorithms for matching and preparing flights for VCSPA are unlikely in the near-term, simple decision support based on existing TMA data may help controllers identify suitable aircraft pairs for VCSPA. ${ }^{46}$ Figure 3 represents a sample TMA timeline available to either the radar controller or the traffic manager; the timeline provides accurate arrival time predictions that are used to manage traffic. A flight's call sign moves down the timeline (closer to arrival) as time progresses. As depicted in Figure 3, TMA's timeline display could include highlighting of aircraft pairs to assist controllers or traffic managers in identifying potential VCSPA pairs. Identifying suitable pairs at an earlier stage (in this case, VRD925 and AAL463) could increase the number of SOIA operations conducted during an arrival rush and reduce average delay. Increased 'marginal' airport capacity would be the primary benefit of increased SOIA operations.

A new scheduling functionality in the NAS is not likely in the near-term. Prior research during CTAS development determined that it was difficult to obtain benefits through improved scheduling optimality in today's air traffic system. ${ }^{7,38,47}$ As long as controllers retain separation authority and the ability to vector aircraft as part of nominal spacing and separation practice, automation will have a difficult 


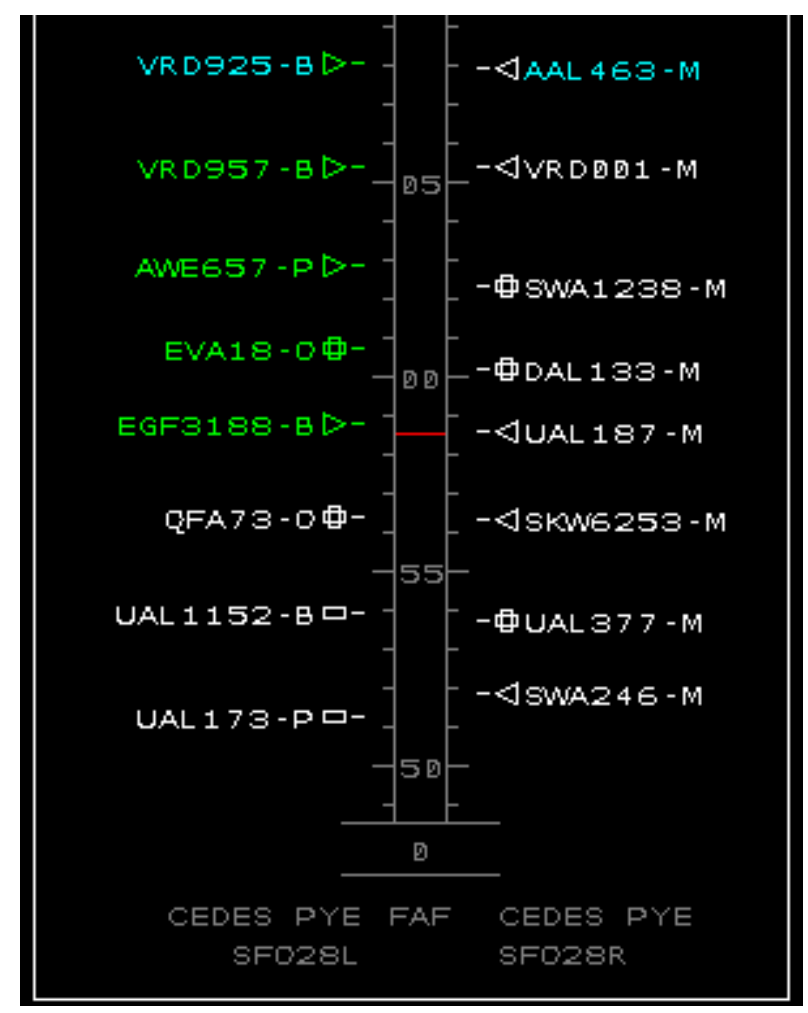

Figure 3: Enhanced TMA display highlighting suitable SOIA pairs in Cyan

time inferring aircraft intent needed for accurate trajectory prediction. For this reason, significant changes to controller roles and responsibilities are likely required for schedule optimization benefits to be realized.

\section{Merging and Spacing}

Methods available for Merging and Spacing of terminal air traffic will remain unchanged. However, to realize efficient descent and climb benefits in the near term, controllers will need to employ a slightly different mix of control than they are accustomed to. RNAV and RNP procedures, if executed as prescribed, do not offer vector (lateral) control as a means of spacing aircraft. While vectors will be an option in the near term to ensure separation, nominal spacing of in-trail and merging arrivals and departures should be conducted with minor adjustments to an aircraft's speed. Situational display aides, such as tiepoint markers, are expected to enhance controller proficiency in spacing aircraft with speed adjustments and serve to eliminate the majority of terminal vectoring on OPD and RNAV/RNP procedures. Merging RNAV/RNP flows with conventional, vectored streams will require a slightly different approach to realize expected OPD benefits. Controllers will necessarily use the conventional flow as a tactical demand buffer, extending or shortening nominal paths to fill arrival and departure slots to accommodate variance in interaircraft spacing on the precision procedures. While spacing delegated to the flight crew shows great promise for future operations, neither the equipage nor the scheduling infrastructure is likely to be in place. Flight Deck Managed Spacing (FDMS) on precision procedures may have difficulty executing prescribed schedules in the presence of wide area differential wind errors. ${ }^{48}$ Therefore, adoption of FDMS may require improvements such as enhanced weather forecasting, extended ADS-B communication and new scheduling functionality, that will not yet be available.

\section{Tactical Separation}

Increased levels of automation and delegation of some functions from the controller to the flight crew (e.g., spacing behind an assigned lead aircraft) could reduce the situational awareness of the controller and impact the controller's ability to identify potential losses of separation in a timely manner. New Tactical Separation capabilities will begin the transition to a stronger dependence on decision support and 
automation to safely manage increased air traffic demand on precision procedures. In the near term, automation will alert controllers of imminent losses of separation between aircraft, while the definition and execution of resolution clearances will remain a responsibility of the controller. Two new capabilities are proposed to begin the transition toward increased dependence on automation for Tactical Separation assurance: the Automated Terminal Proximity Alert system (ATPA) and an enhanced tactical conflict prediction algorithm ${ }^{49,50}$

These capabilities are proposed to supplement or replace the legacy Conflict Alert system in TRACON facilities. Figure 4 shows a prototype Tactical Separation assurance display for terminal airspace. The ATPA cone depicts the required separation between aircraft along the direction of flight. An orange ATPA cone indicates an 'Immediate Attention Alert,' as differentiated from a 'Cautionary Alert,' which would be displayed in yellow and indicate less time criticality. An audible warning would draw the controller's attention to both types of alert. In the near-term, only alerting functionality would be provided, and the controller would define and issue a resolution clearance to one or both aircraft involved. In Figure 4 , aircraft positions are identified with an " $\mathrm{H}$ " and are landing from left to right. Tang has developed a preliminary conflict prediction algorithm that demonstrates how improved intent inference techniques can increase alert lead-time without adversely impacting false alert rates as compared to the legacy Conflict Alert system. ${ }^{50}$ Accurate intent modeling is critical to any conflict prediction function; the inference techniques developed by Tang, along with increased use of precision procedures, will enable automation systems to provide timely warning of loss of separation.

ATPA is being developed by the FAA to alert controllers of imminent loss of required spacing between aircraft on final approach and builds upon the capabilities of the Terminal Proximity Alert (TPA). Like RNAV/RNP procedures, IAPs provide accurate lateral intent. Further, IAPs provide accurate vertical intent as well once aircraft are established on final approach. However, aircraft speed and configuration for landing has been shown to be critical to predicting accurate arrival times, and thus to predicting interarrival spacing on final approach. Gong has developed an empirical mechanism that may improve speed intent modeling and final approach trajectory prediction in tools such as TPA. ${ }^{51}$ It is anticipated that such modeling will be necessary to improve ATPA functionality over Conflict Alert and TPA. ATPA may be integrated with the improved conflict prediction algorithm to provide continuous alerting coverage throughout TRACON airspace. ${ }^{52}$

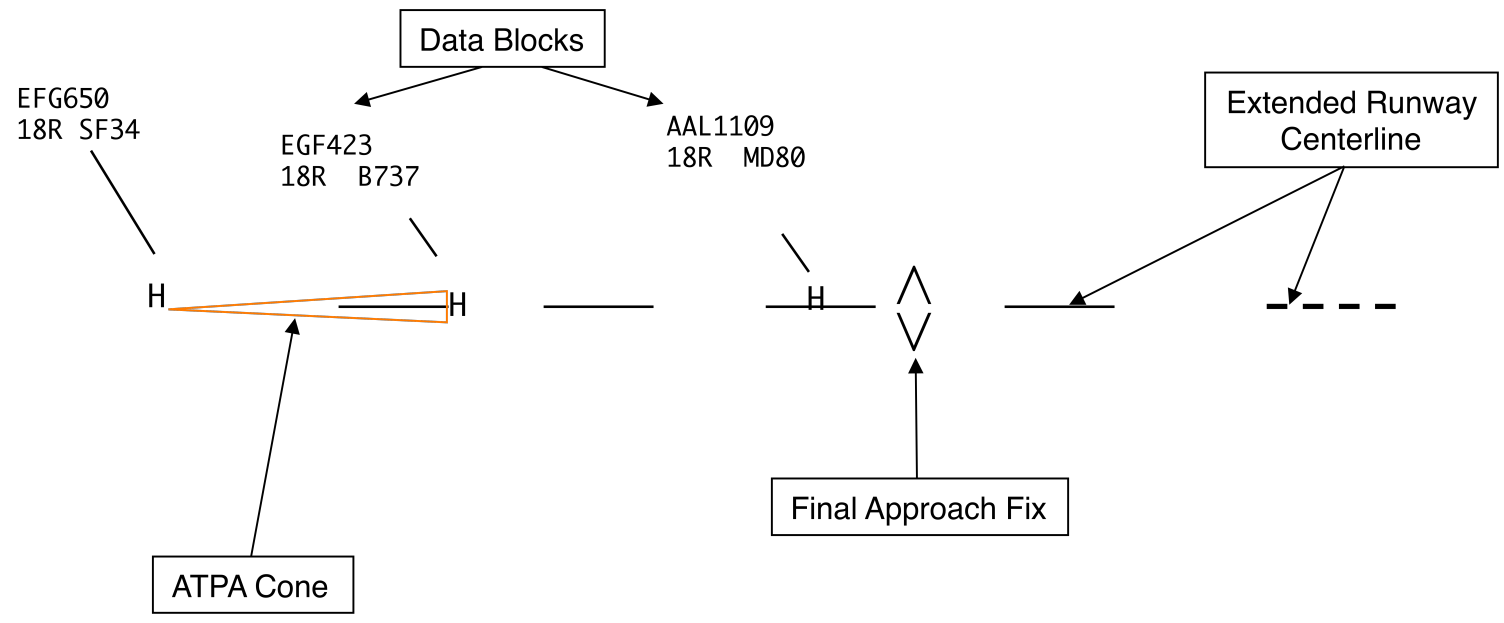

Figure 4: Prototype Display for Tactical Separation Assurance in Terminal Airspace

\section{Off- Nominal Recovery}

For Off-Nominal Recovery to function properly, nominal operations must first be defined such that non-conformance can be detected. Near-term operations will include many flights for which only the controller knows the definition of nominal behavior. Thus, no changes to existing procedures are expected for recovery from off-nominal events and operations. With the exception of the proposed Tactical Separation alerting functions, identification of off-nominal events and conditions will remain with those currently responsible (i.e., traffic managers, controllers and flight crews). Clearances that provide for 
temporary safe operation of affected aircraft will be defined by the controller and/or flight crew, as will clearances to return aircraft to nominal operations or manage emergency aircraft.

\section{Mid-Term SDO Capabilities}

1. Assumptions for the Mid-Term

Mid-term SDO will introduce significant changes to terminal operations. Fundamental shifts in CNS technology will begin to occur, building on OPD and RNAV procedures and depending on increasing numbers of aircraft equipped for RNP procedures and with data communications. The following assumptions are made regarding terminal operations in the mid-term:

- RNAV/RNP routes will define the complete lateral path in the TRACON; STARs and SIDs may additionally include altitude and speed restrictions.

- Aircraft will be controlled to meet STAs at the runway threshold and at key merge points.

- The controller will be responsible for aircraft separation with DSTs to aid efficient management of traffic.

- Some aircraft will be equipped for Flight Deck Managed Spacing, and such operations will be permitted at the controller's discretion.

- Communications between the flight crew and air traffic will be via voice and data communication (for equipped aircraft).

\section{Extended Terminal Area Routing}

Extended Terminal Area Routing will select from a predefined set of RNAV/RNP procedures. Automation will select the assigned procedure for a flight based on airport configuration, traffic demand, convective weather and other airspace constraints (e.g., SUA or MOA). Arrivals will consist predominantly of OPDs except for aircraft not sufficiently equipped (lacking a Flight Management System), and possibly excluding some OPDs during peak demand periods. The controller will issue arrival and departure clearances consistent with the selected RNP procedure either via voice or data communication for equipped aircraft.

The mid-term extended routing function is impacted by three constraints: 1) the slow adoption of data communications, 2) the expected difficulty of controller-managed separation on dynamic (i.e. defined in real-time) RNP routes, and 3) the lack of appropriate certification processes for dynamic RNP routes. Controller-managed Merging and Spacing with speed adjustments at significantly increased traffic levels (compared to today) is a difficult task. The predefined set of procedures will provide the repeatability controllers need to develop effective speed-based spacing techniques for RNP procedures. Perhaps more importantly, data communication will not be widespread, likely making dynamic RNP procedures impractical due to voice frequency congestion. While truly dynamic RNP procedures in the mid-term are unlikely, flexibility in terminal routing remains important to manage some merging geometries and to provide buffers for off-nominal events.

RNAV/RNP will allow for closer spacing of flows into terminal airspace; increasing the number of available arrival routes will allow for rerouting flows of aircraft when blockages limit the use of nominal arrival routes. The primary objective of multiple RNAV/RNP arrival routes is to provide the Extended Terminal Area Routing function flexibility to mitigate airspace blockage and demand fluctuations. The RNP requirements can be tailored to meet the demand set capabilities (e.g. if some aircraft can meet RNP4, but not RNP1), or for weather constraint mitigation. In such instances, the extended terminal area routing function would assign aircraft to flows compatible with their capabilities. Figure 5 shows a sample RNP route structure for a typical arrival pattern inside terminal airspace. A typical arrival pattern consists of a primary RNP arrival procedure from each metering fix to each allowable landing runway. Secondary and tertiary variations of each primary arrival procedure are defined, allowing for increased or decreased terminal flight time. The automation selects a procedure appropriate to the runway demand, and provides the controller with a precisely defined routing option to mitigate larger perturbations and off-nominal events. These multiple routes provide a demand buffer that is used by scheduling to mitigate uncertainty that speed control along the route cannot. Lastly, some of the functionalities outlined by the FAA's 'Big Airspace' concept will be introduced: dynamically configured airspace between arrival and departure operations, extended use of diverging course procedures, and expansion of airspace requiring three nautical miles of lateral separation. ${ }^{53}$ 


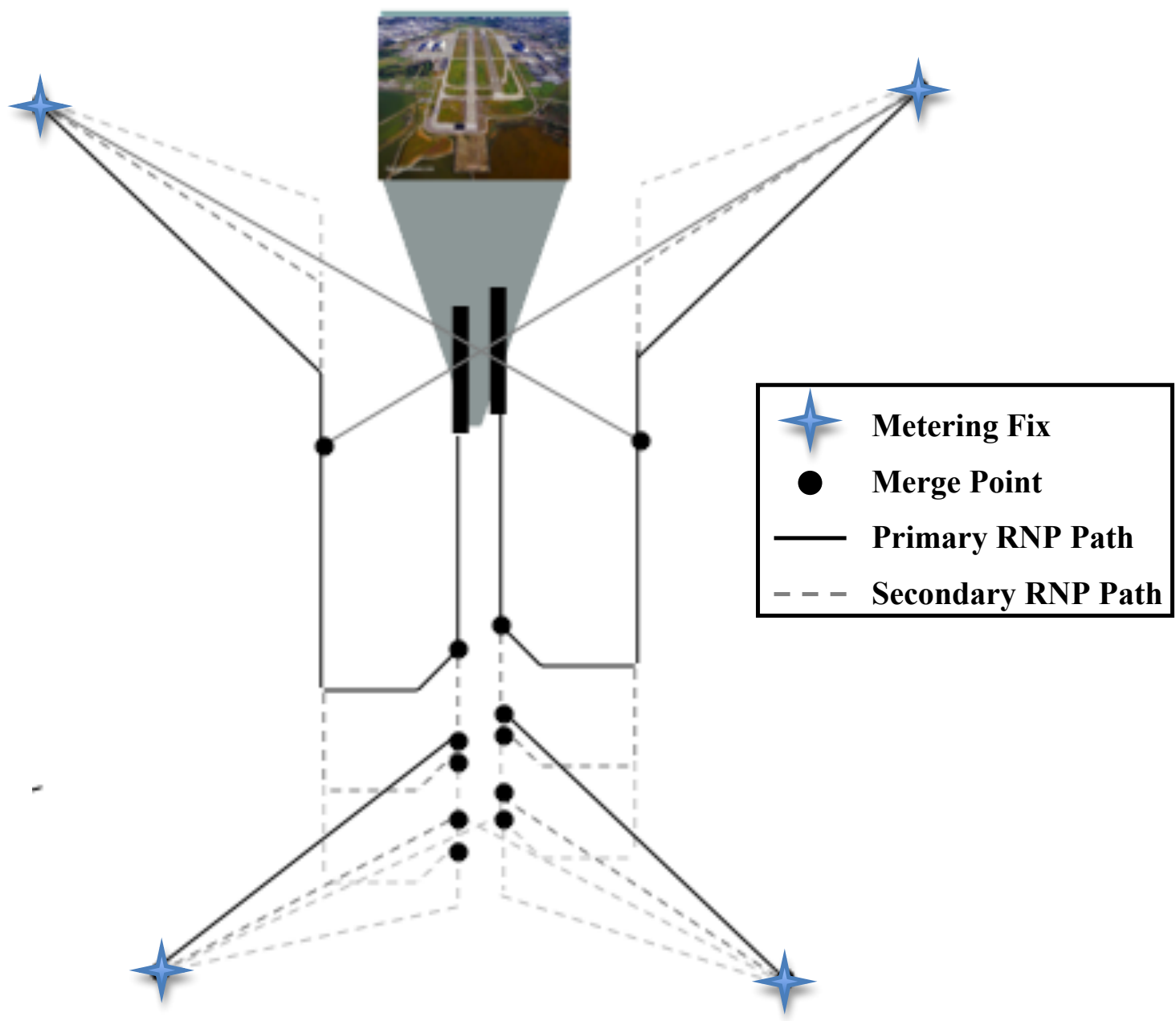

Figure 5: Typical Arrival Pattern with Multiple RNP Arrival Paths

Recent work by Thippavong et al. suggests this type of flexible routing framework is needed to enable a schedule to be defined using only speed control for Merging and Spacing, while limiting the need for spacing buffers on final approach and at departure metering fixes. ${ }^{54}$ The use of demand buffers in the form of multiple terminal route options will enable greater numbers of OPDs, replacing demand buffers in the form of level powered flight with descending, albeit (sometimes) longer paths. While elimination of level, powered flight at lower altitudes is often viewed as the primary goal of procedural and scheduling improvements in NextGen, analyses by Robinson suggest reducing delay is a more important factor during peak arrival periods when path stretching is needed for metering or aircraft separation. ${ }^{44}$ While the Extended Terminal Area Routing function will provide RNAV/RNP procedures with the flexibility necessary to mitigate inherent uncertainties, significant fuel savings compared to today's terminal buffering technique will rely on scheduling improvements targeting delay reduction.

\section{3. $\quad$ Precision Scheduling Along Routes}

The mid-term scheduling function will utilize significantly improved trajectory predictions, but is highly constrained in the flexibility most aircraft will have to mitigate expected operational variances (e.g. wind forecast errors, pilot and controller delays, or variations in FMS behavior ${ }^{55}$ ). The scheduling function 
will need to consider the impact of limited flexibility associated with RNP procedures in the context of nominal aircraft and system performance. With multiple RNP-defined paths available for each aircraft, the scheduler will employ path adjustments (selecting different landing pattern paths from Figure 5) to match demand with capacity, and speed adjustments to fine-tune inter-aircraft spacing. Many aircraft will have the ability to control to a single Required Time of Arrival (RTA) along its trajectory. Time of arrival control will require the scheduler to consider aircraft RTA capability, including constraints on when an RTA can be assigned to the flight crew, and what aircraft-specific constraints are applied once an aircraft is pursuing an RTA.

RNP procedures provide improved flight path predictability at the expense of constraining interaircraft spacing to primarily speed adjustments. This places additional onus on the scheduling function to order aircraft in a manner that ensures the prescribed sequences along an aircraft's route are feasible within the speed control of the aircraft and with required separation between aircraft. This is a noteworthy departure from existing scheduling constructs in that tools, such as TMA, only address feasibility of STAs and do not guarantee that separation with other aircraft along the path prior to the STA is feasible. TMA depends on the controller to define a feasible path to meet the STA, and to override the advisory when the STA would require unreasonable workload. The scheduling capability will evaluate separation at key points along the path of each aircraft (e.g., merge points) and not just at runways and metering fixes. Failure to do so will result in controller interventions that negate the benefits of efficient procedures and reduces the flight path predictability needed for the Tactical Separation function.

A new scheduler is under development at the NASA Ames Research Center that extends TMA scheduling functionality to consider merges in arriving flows and constrains the schedule to sequences that are feasible at all merge points within the defined speed limits for each aircraft and the procedures being flown. Additional enhancements under development to increase the efficiency of scheduled RNP operations include time advance, time recovery and constrained position shifting (CPS). Time advance was proposed by Erzberger; it was found that by accelerating aircraft near the beginning of an arrival rush, overall delay and fuel burn was significantly reduced. ${ }^{56}$ Time recovery refers to closing gaps in a schedule that have developed due to inherent operational variances, either by accelerating aircraft or delaying planned speed reductions. ${ }^{54}$ CPS was first proposed by Dear $^{37}$ and was evaluated as part of TMA development. ${ }^{38}$ CPS was subsequently removed from the TMA scheduling capability when simulation evaluations showed it resulted in unacceptable controller workload because of variation from the standard First-Come-First-Served processes. Simulation evaluations are needed to determine if merge point scheduling with enhanced decision support can enable CPS at acceptable controller workload levels. Time advance and time recovery will require significant interaction between the flight crew and the controller.

RTAs will be reviewed and assigned by controllers by voice or data communication. As will be discussed in the next section, controllers will have to manually space aircraft not equipped for data communications by issuing speed clearances along the path. Because the controllers remain responsible for separation along these paths, controller familiarity and proficiency with TBFM will be critical to SDO. Lack of proficiency could lead to increased controller intervention rates, reduce schedule stability and negate some of the benefits of efficient descent and climb procedures. Additionally, the controller is expected to provide the flight crew with expected runway assignment and associated merge point times of arrival. In the event that the schedule becomes infeasible or undesirable, the Traffic Management Coordinator (TMC) will initiate a reschedule for all or a subset of aircraft. Coordination between facilities will be managed by the Traffic Management Unit (TMU), and will be relatively unchanged from today's processes.

Precision Scheduling Along Routes will consider all aircraft in the extended terminal airspace, but will not integrate surface or en route operations in the schedule. Interaction with surface movement and en route constraints (e.g., traffic management initiatives) will be treated as a boundary condition or constraint to the scheduling problem. Airports will dictate the configurations that define which runways are active and what procedures are available for their use. For example, the scheduler would need to consider aircraft RNP capability when airports are conducting RNP-based closely spaced approaches. Further, any constraints on operations such as arrival rates, spacing exceeding minimums, blocked slots, and priority flights will also be provided as inputs to the SDO scheduling function.

\section{Merging and Spacing}

The Merging and Spacing function controls aircraft that are nominally navigating precision procedures. The scheduling function provides order to arriving and departing aircraft under the constraints 
of aircraft performance and procedural restrictions. This provides the Merging and Spacing function with accurate predictions of future aircraft position. Two mechanisms for Merging and Spacing will be at the controller's disposal: Flight Deck Managed Spacing (FDMS) and Controller Managed Spacing (CMS).

CMS is similar in function to near-term Merging and Spacing, but with the addition of key decision support. Under CMS, ground-based automation will provide the controller with decision support in the form of enhanced spacing and advisory cues to meet spacing requirements and scheduled times of arrival. ${ }^{57}$ Development of the active Final Approach Spacing Tool (aFAST), which extended pFAST by providing controllers with turn and speed advisories, demonstrated the complexity of providing controllers with automated control advisories while the controller retained separation responsibility. ${ }^{47,58}$ Simulation evaluation will investigate if CMS can overcome this difficulty through application of RNP procedures and improved decision support or if it will simply provide enhanced spacing information to better inform the controller's own spacing technique. Under CMS, the controller will issue speed clearances via voice or data communication. The ground-based automation will also assist the controller in identifying sequence and spacing for FDMS equipped flights.

FDMS was developed on multiple fronts and is used here to refer generically, to inter-aircraft spacing as managed by the flight crew with the assistance of flight deck decision support. ${ }^{59}$ Varying levels of FDMS capability and decision support have been developed; a number of systems will likely be available in the mid-term, but few aircraft will be so equipped. FDMS systems utilize precision surveillance (ADS-B) and knowledge of ownship (trailing) and dependent (lead) aircraft intended trajectory to provide spacing guidance to the flight crew. FDMS has been shown effective in managing inter-aircraft spacing on final approach as well as for spacing aircraft on efficient descents with increased spacing. ${ }^{33}$ Earlier FDMS systems provided spacing guidance based on a predicted direct flight to a future waypoint, while recent efforts rely on GPS-based procedures and are able to provide more accurate guidance with the improved intent knowledge. The controller will be responsible for Merging and Spacing of aircraft, but will have the authority to delegate that responsibility to the flight crew, indicating an aircraft-to-follow and an assigned spacing. The flight crew accepts separation responsibility with the assigned lead aircraft to avoid the aforementioned authority/control dilemma of aFAST. Separation responsibility with all other aircraft remains with the controller, but with additional Tactical Separation decision support.

\section{Tactical Separation}

As new technologies and procedures are introduced into SDO, the Tactical Separation function will continue to provide a safety net independent of the Merging and Spacing control function. The Tactical Separation function will build on the mid-term conflict alerting functionality; controllers will not only be alerted of imminent loss of separation, they will be provided with tools to assist in resolving the pending conflict. The prevalence of RNP procedures will improve the performance of the conflict prediction algorithm by providing accurate lateral and (to a lesser extent) vertical intent for all nominal operations. Compared to the near-term, the conflict prediction algorithm will provide increased alert lead times and/or a reduced false alert rate. Improved heuristics will be developed to identify flights that are not conforming to their prescribed trajectory, further improving alerting performance. The controller will be alerted of imminent loss of separation and immediately provided with a two-minute conflict-free resolution for one or more aircraft involved. The controller has the option to accept the provided resolution or to define a new resolution. If the controller chooses to define his or her own resolution, terminal automation may provide a trial-planning functionality to assist the controller in defining a short-term conflict-free resolution. ${ }^{52}$ In all cases, the resolution will be issued as a series of clearances via voice to the flight crew, and entered into the terminal automation system (to maintain accurate flight intent). However, Erzberger proposes that the tactical separation assurance function might be nearly automated by utilizing mostly existing hardware (Mode-S, TCAS) and aurally annunciating resolution maneuvers on the flight deck. ${ }^{60}$ The short-term conflict-free path cleared by the controller is intended to provide the controller with the time necessary to evaluate the situation and determine a course of action in coordination with the flight crew and other controllers (if necessary).

\section{Off- Nominal Recovery}

Off-Nominal Recovery will remain a human-centric function. The TMU, controllers and flight crews will coordinate return to nominal operations. The TMU will have the authority to initiate a reschedule of operations (system-wide or local), and can place a blocked slot in the schedule in coordination with other controllers. The controllers will have a trial planning functionality similar to that 
provided for Tactical Separation to assist in defining conflict-free return paths into the desired traffic flow. Once defined, return paths will be cleared via data communication or voice and entered into the terminal automation system to maintain accurate flight intent. In addition to alternate RNP procedures defined by the Extended Terminal Area Routing function, planned buffers will be included to assist the controller in managing larger scale disturbances or capacity fluctuations.

Krozel et al. proposed the concept of 'wiggle room;' buffers placed near or adjacent to precision procedures that provide the airspace flexibility needed to effectively mitigate off-nominal events. ${ }^{61}$ Where uncertainty in weather forecasts impact RNP route availability, controllers could, within the confines of the planned 'wiggle room,' direct aircraft on a return path to nominal operations without concern for conflicting with other RNP procedures. Krozel also proposed planned demand buffers just outside terminal airspace as a means to mitigate the impact of capacity fluctuations due to weather forecast uncertainty. ${ }^{62}$ Limited demand buffers in the form of holding patterns were shown to significantly reduce system-wide delay compared to more traditional methods.

\section{E. End-State SDO Capabilities}

\section{Assumptions for the End-State}

End-State operations are characterized by a substantially different role for the air traffic controller. For most routine operations, the controller will not be involved in the generation or transmission of clearances; separation responsibility will rest elsewhere as well. The following assumptions are made regarding end-state SDO:

- $3 \mathrm{D}$ and/or 4D RNP routes within the TRACON will be defined in terms of latitude, longitude, altitude and (in some cases) nominal speed along the entire route.

- Aircraft will be controlled to meet scheduled times of arrival at the runway threshold, merge points and where coordination is required between flows.

- Generation, transmission and execution of clearances for routine operations will be automated, requiring only flight crew acceptance.

- Some aircraft will be equipped for FDMS, and such operations will be assigned and cleared by automation.

- DSTs will aid the controller in efficient management of non-equipped aircraft and off-nominal events.

- Communications between the flight crew and air traffic will be via voice and data communication, with routine clearances being issued via data communication.

\section{2. $\quad$ Extended Terminal Area Routing}

End-State SDO will rely on data communications to relay RNP procedures to the flight deck for loading into the FMS. As such, the form of these routes need not be overly simplistic or even predefined. Extended Terminal Area Routing will be dynamic in accounting for traffic demand, weather phenomena (convection, icing, turbulence, etc.) and airport configurations. While IAPs and the initial portion of SIDs will remain static, and repeated patterns in TRACON airspace will provide a backbone for traffic flows, 4D trajectories connecting en route flight with the runway will make use of available airspace in defining the efficient paths considering real-time constraints.

All arrivals will be conducting OPDs under nominal operations. RNP departures will be utilized as well and will introduce new levels of vertical efficiency by coordinating flows between arrivals and departures, and between metroplex airports. For dynamic routes to be realized and executable, a reliable mechanism for generating RNP routes in real time needs to be developed that meets all applicable standards (e.g., Terminal Instrument Procedures or Special Aircraft and Aircrew Authorization Required). If such a capability is developed, the dynamic weather avoidance routes can be defined to leverage the RNP capabilities of a given demand set. That is, aircraft of mixed RNP capabilities will be assigned to routes that maximize the efficiency or throughput of operations depending on the traffic demand. Figure 6 shows an example weather impacted terminal area with mixed-RNP routes designed to meet the traffic demand while avoiding weather constraints. Dynamic RNP routes will follow a predictable pattern in the TRACON, but these nominal routes will shift to avoid weather (e.g., by varying the downwind flight segment offset from the airport) and will place additional restrictions on the RNP capability required for navigation of each route. The routes in Figure 6 are displaced from nominal STARS to avoid convection; the varying width of the routes indicate different levels of RNP equipage required to fly each route. 


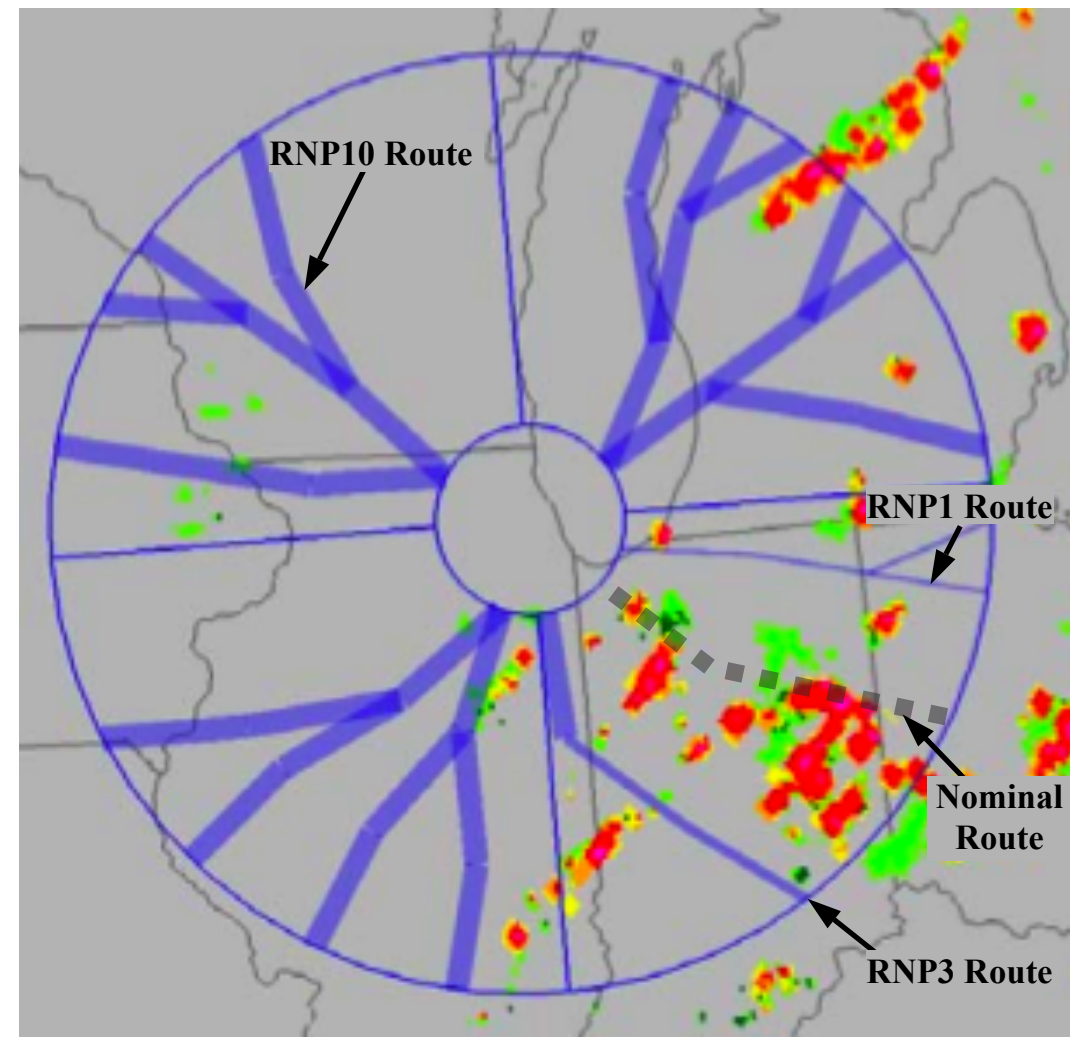

Figure 6: Dynamic RNP routes in weather-impacted airspace

(Image courtesy of: J. Krozel, Metron Aviation)

Because weather forecast accuracy improves with reduced look-ahead, once aircraft enter extended terminal airspace, weather forecasts become much less stochastic and are treated as deterministic for planning purposes. Ongoing research indicates that while a pilot's willingness to penetrate convective weather in terminal airspace is difficult to predict, route availability is not. ${ }^{30}$ That is, given a finite variance on route length, it has been shown to be possible to predict with a high degree of certainty that a given route will be available for an aircraft to navigate through/around convective weather in the terminal area. Future research needs to determine if mitigation of other weather impacts (e.g., turbulence) can be achieved in a similar fashion.

Some arrival routes may only be available to better-equipped aircraft, especially at peak demand times. While the vision of dynamic RNP operations provides a framework for shuttling aircraft quickly into and out of terminal areas and efficiently around weather impacted airspace, assigning aircraft to such routes and ordering aircraft on these routes is a daunting challenge for the scheduling function. If improved forecasting products fail to materialize, other mechanisms such as down linked flight intent from the flight deck will need to be explored.

\section{Precision Scheduling Along Routes}

Precision Scheduling Along Routes will build on the mid-term capability by adding automatic transmission of scheduled times, automatic identification and assignment of paired SOIA and VCSPA operations, and automatic rescheduling as needed. While the TMC will retain the ability to initiate a reschedule at any time, generation and transmission of SDO schedules will be automated. Dynamic RNP routes also place additional constraints on the SDO scheduler.

Route assignment becomes dependent on aircraft equipage in much the same manner as fieldlength limitations constrain runway selection; aircraft can only be assigned procedures for which they are capable of navigating under the specified RNP level. Under nominal conditions, precision RNAV/RNP procedures will be used for all aircraft operations. Data communication of scheduled times at merge points and the runway are key to execution of precision procedures under high traffic density. Time-of-arrival control will utilize multiple RTA capability when aircraft are so equipped. While enhanced weather 
forecast products will allow for generation of routes more likely to mitigate weather constraints, the ability of the schedule to effectively assign aircraft on these routes depend on their availability as predicted, and therefore on the weather forecast products themselves. Lastly, the scheduler will expand on merge point scheduling to enable scheduling to dynamic merge points and to allow coordination of aircraft flows not sharing runway or departure airspace resources (e.g., crossing arrivals and departures or metroplex traffic).

The end state of SDO in the NextGen timeframe may not require integrated scheduling, but will (at a minimum) include coordination of metroplex and arrival/departure flows. Coordination between arrival and departure schedules, and between airports in a metroplex will allow efficient sharing of airspace where appropriate. However, integrated airspace for scheduled operations is not expected to be prevalent in SDO. Capozzi showed integration may have only limited benefit in metroplex airspace, and can be equally well served by procedural segregation and limited coordination. ${ }^{23}$ Only in cases of substantial inefficiency will integrated scheduling be pursued.

\section{Merging and Spacing}

As in the near-term and mid-term phases, the end-state Merging and Spacing function serves as the inner-loop control of SDO operations: generating, communicating and executing minor adjustments to the cleared procedures resulting from Extended Terminal Area Routing and Precision Scheduling Along Routes. However, the role of the controller in this process is much less clear. FDMS equipage will be relatively common, but not required. Data communication may be required for access to SDO airspace (arrival or departure); this does not indicate airports will be inaccessible; such restrictions may only be in place during peak traffic periods. Significant changes are expected for both airborne and ground-based variants of the Merging and Spacing function.

In Ground-Based Merging and Spacing (GBMS), speed clearances will be automatically generated and transmitted to aircraft via data communication. Confirmation of clearance acceptance will be logged by ground automation and will revise aircraft intent. In this framework, automation systems will necessarily have authority and responsibility for aircraft spacing. The controller or ground automation will have the authority to delegate Merging and Spacing to FDMS-equipped aircraft.

FDMS-equipped aircraft that have accepted spacing responsibility will operate in much the same manner as in the mid-term; spacing and aircraft-to-follow will be assigned via data communication from the ground automation. The flight crew will also assume separation responsibility with the assigned lead aircraft. Dynamic routes will require that intent information of the lead aircraft be communicated to the trailing, spacing aircraft. Data communications between aircraft are needed since no database of dynamically created RNP procedures will be available to the onboard systems that provide intent of the lead aircraft. IAPs may include all-weather VCSPA procedures. ${ }^{63}$ VCSPA procedures assigned by the scheduling function as a paired approach will require significant enhancement to the onboard spacing capability. Such operations will likely require coupling of the autopilots for spacing via a high-speed data link that includes roll angle. Both FDMS and automated GBMS fundamentally change the role of the controller; it is unclear if the controller can or will retain separation responsibility without explicit spacing control.

\section{Tactical Separation}

It is highly controversial to propose that controllers be relieved of separation responsibility. However, few will dispute that for a given sector/position, there exists a limit of air traffic demand exceeding the controller's ability to provide safe separation. Controller aids, enhanced information, decision support and flight deck delegation will all serve to increase that safe limit, but a finite capacity limit remains for any given concept. A thorough study of system/controller response to off-nominal situations is necessary to identify controller spacing and separation limits. An incremental approach is necessary, not only to foster a smooth transition to a substantially different approach to air traffic control, but also to ensure the safety of the system is not compromised; each layer of the safety mechanism must be validated and a measured implementation will allow for rational assessment of each element. Practical limitations of controller provision for separation in the NextGen timeframe will need to be identified to determine an appropriate balance of roles and responsibilities between automation, flight crew and air traffic controllers. Independent of separation responsibility, it is likely such an automated system would adversely impact the controller's situational awareness. Near-term aircraft trajectory and separation predictions are likely to be significantly more difficult for the controller given the dynamic nature of 
terminal procedures. The Tactical Separation function becomes a critical safety net given the potential for compromised controller situational awareness and predictive ability.

The Tactical Separation function will provide an additional safety layer between TCAS and the controller; or, in the event controller separation responsibility is untenable, will become a critical element of automatic aircraft separation provision. The Tactical Separation function will comprise three capabilities: 1) prediction of imminent loss of separation, 2) automatic generation of two-minute conflictfree resolution, and 3) automatic transmission of resolution (in most cases). ${ }^{52}$ An improved conflict prediction algorithm will form the basis of the prediction and resolution generation, sampling multiple possible resolutions for conflicts within the two-minute look-ahead period. The conflict-free resolutions will be transmitted to the flight deck via data communication for most aircraft. To be employed in this fashion, aircraft will require systems that allow for rapid execution of clearances issued via data communication. The controller will advise unequipped aircraft via voice. The Tactical Separation functions will be independent of other SDO functions and will integrate seamlessly with collision avoidance systems. As in the mid-term, the Tactical Separation resolution advisories are intended to buy time for the controller to coordinate with flight crews and other controllers to diagnose the situation and initiate Off-Nominal Recovery.

\section{Off- Nominal Recovery}

The end-state will bring enhanced decision support to the Off-Nominal Recovery function while retaining the central role of the controller in evaluation, coordination and planning. Even in the highly automated SDO, it will remain difficult to identify and adapt to all causes of non-conformance. Human creativity and complex problem solving ability will likely be required in many off-nominal scenarios. The ability of the controller to manage Off-Nominal Recovery in a timely fashion will require a number of new tools and technologies.

Decision support for the controller will be enhanced to include rapid constraint input. For example, 'NDL' entered into the controller scratchpad could indicate 'no data link,' constraining solutions to those which can be effectively cleared via voice. As constraints are identified, the controller will be presented with potential solutions for review. Each solution will indicate a reinsertion sequence and a return path. Alternatively, a trial planning functionality will be at the controller's disposal to form his or her own solution, which will be checked for conflicts and against all known constraints for feasibility. Upon controller approval, the resolution(s) will be transmitted to the flight deck via data communication, or by voice if required. For aircraft unable to receive data communication clearances, the controller may issue a clearance via voice. Recovery from off-nominal events is often subject to a myriad of constraints, both related and unrelated to the event itself. Decision support must provide to the controller timely information relevant to a very dynamic context.

For mitigation of routes blocked by convective weather (one instance of off-nominal operations), Optimal Path Maps (OPMs) have been proposed for all aircraft as they proceed along their trajectory. ${ }^{25}$ OPMs would at every instance in time provide a number of feasible routing options meeting all known constraints. Controllers could use OPMs to either directly select a solution from the set, or to trial plan a solution from one of the OPM-defined templates (e.g., dragging a waypoint or eliminating an altitude restriction). By inputting additional constraints into the Off-Nominal Recovery function, the OPM would be pruned to only those paths that meet all constraints. SDO is envisioned to utilize OPMs as a database of Off-Nominal Recovery options that will be leveraged by the controller in defining a safe return to nominal operations. Therefore, one path in the OPM must always be the 'emergency' or 'priority' path that ignores all efficiency constraints in favor of expeditious resolution (e.g., landing at the nearest airport). 


\section{Concluding Remarks}

This paper has presented a concept for future terminal air traffic operations with a focus on robustness to inherent systemic uncertainties and realistic transition to end-state capabilities. A terminal air traffic operations functional architecture has been defined which describes five enduring functions: 1) Extended Terminal Area Routing, 2) Precision Scheduling Along Routes, 3) Merging and Spacing, 4) Tactical Separation and 5) Off-Nominal Recovery. The capabilities within each functional element are detailed for near-term, mid-term, and end-state NextGen phases. An attempt has been made to document the myriad of supporting literature for each capability phasing decision, but much remains unknown regarding effective roles and responsibilities in the mid-term and end-state. For this reason, a series of human-in-the-loop (HITL) simulations and fast-time analyses will be conducted to refine the concept of operations.

Researchers at NASA's Ames Research Center and Langley Research Center have defined a progression of simulations to address the remaining task delegation questions and to develop supporting algorithms and procedures. Building on simulations for TMA, FAST, EDA and FDMS capabilities, current and planned simulations will evaluate many of the mid-term and end-state concept elements described in this paper. To ensure an effective transfer of research findings into system requirements, close involvement with the FAA and the JPDO is required.

The FAA and NASA have convened a set of Research Transition Teams (RTTs) to ensure the effective transition of NASA concepts and technologies to the FAA for NextGen implementation decisions. Most of NASA's terminal air traffic research activities relate to the Efficient Flows Into Congested Airspace (EFICA) RTT. The EFICA RTT is concerned with Trajectory Management functions of the NextGen concept in congested airspace. The SDO concept presented in this paper identifies and begins to address a number of foundational mid-term requirements for NextGen to be successful. The TBFM Concept Engineering and Operational Evolution Plan details a number of synergies between the SDO midterm capability set and NextGen Operational Improvements. ${ }^{64}$ NASA will continue to work with FAA through the EFICA RTT to identify areas of effective collaboration and to inform RTT activities with relevant research results.

\section{References}

1. "Concept of Operations for the Next Generation Air Transportation System," Ver. 3.0, Joint Planning and Development Office (JPDO), Washington, D.C., Oct. 2009.

2. Fitts P. M., "Human engineering for an effective air navigation and traffic control system," National Research Council, Washington, D.C., 1951.

3. Kirlik, A. Adaptive perspectives on human-technology interaction: Methods and models for cognitive engineering and human-computer interaction, Oxford University Press, New York, 2006.

4. Parasuraman, R., Riley, V. "Humans and Automation: Use, Misuse, Disuse, Abuse," Human Factors, 1997, 39(2), pp 230-253.

5. Sierhuis, M, Bradshaw, J. M., Acquisti, A., van Hoof, R., Jeffers, R. \& A. Uszok. "Human-agent teamwork and adjustable autonomy in practice," Proceedings of the 7th International Symposium on Artificial Intelligence, Robotics, and Automation in Space: I-SAIRAS, Nara, Japan, 2003.

6. "National Airspace System: Free Flight Tools Show Promise, but Implementation Challenges Remain," General Accounting Office (GAO) Report to Congressional Requesters, GAO-01-932, Washington, D.C, August 2001.

7. Swenson, H. N., Hoang, T., Engelland, S., Vincent, D., Sanders, T., Sanford, B., and Heere, K., "Design and Operational Evaluation of the Traffic Management Advisor at the Fort Worth Air Route Traffic Control Center," 1st USA/Europe Air Traffic Management R\&D Seminar, Saclay, France, June 1997.

8. Green, S. M., and Vivona, R., "Field Evaluation of Descent Advisor Trajectory Prediction Accuracy," AIAA-963764, AIAA Guidance, Navigation, and Control Conference, July 1996.

9. Coppenbarger, R. A., Lanier, R., Sweet, D., and Dorsky, S., "Design and Development of the En Route Descent Advisor (EDA) for Conflict-Free Arrival Metering," AIAA-2004-4875, AIAA Guidance, Navigation, and Control Conference, Providence, RI, 16-19 Aug. 2004.

10. Coppenbarger, R. A., Mead, R. W., and Sweet, D. N., "Field Evaluation of the Tailored Arrivals Concept for Datalink-Enabled Continuous Descent Approach," AIAA-2007-7778, AIAA Aviation Technology, Integration and Operations Conference (ATIO), Belfast, Northern Ireland, 18-20 Sep. 2007.

11. Lee, K. K., and Davis, T. J., "The Development of the Final Approach Spacing Tool (FAST): A Cooperative Controller-Engineer Design Approach," NASA TM-110359, Aug. 1995 and Proceedings of the 5th IFAC Symposium on Automated Systems Based on Human Skill, Sep. 1995. 
12. Davis, T. J., Isaacson, D. R., Robinson III, J. E., den Braven, W., Lee, K. K., and Sanford, B., "Operational Field Test Results of the Passive Final Approach Spacing Tool," IFAC 8th Symposium on Transportation Systems, Chania, Greece, June 1997.

13. Hoang, T., Farley, T., Foster, J., and Davis, T., "The Multi-Center TMA System Architecture and Its Impact on Inter-Facility Collaboration," AIAA Aircraft Technology, Integration and Operations (ATIO) Conference, Los Angeles, CA, 1-3 Oct. 2002.

14. Farley, T. C., Landry, S. J., Hoang, T., Nickelson, M., Levin, K. M., Rowe, D., and Welch, J. D., "Multi-Center Traffic Management Advisor: Operational Test Results," AIAA-2005-7300, Proceedings of the 5th AIAA Aviation Technology, Integration, and Operations (ATIO) Conference, Arlington, VA, 26-28 Sep. 2005.

15. Jung, Y. C., and Isaacson, D. R., "Design Concept and Development Plan of the Expedite Departure Path (EDP)," AIAA Aircraft Technology, Integration, and Operations (ATIO) Conference, Los Angeles, CA, 1-3 Oct. 2002.

16. Williams, D.H., Osequera-Lohr, R.M., Lewis, E.T., "Design and Testing of a Low Noise Flight Guidance Concept," NASA/TM-2004-213516, 2004.

17. Coppenbarger, R. A., Mead, R. W., and Sweet, D. N., "Field Evaluation of the Tailored Arrivals Concept for Datalink-Enabled Continuous Descent Approach," AIAA-2007-7778, AIAA Aviation Technology, Integration and Operations Conference (ATIO), Belfast, Northern Ireland, 18-20 Sep. 2007.

18. "Continuous Descent Operations (CDO)," ICAO PBN Program Office, Workshop on the Development of a Performance-Based Navigation (PBN) Airspace Concept for the CAR Region- Special Implementation Project for the CAR Region, ICAO Doc 9931, Mexico City, Mexico, 5-7 July 2010.

19. Brooks, J., "Continuous Descent Arrivals," ICAO Workshop on Aviation Operational Measures for Fuel and Emissions Reductions. Montreal, Canada, 20-21 September 2006.

20. Becher, T. A., Barker, D.R., Smith, A.P., "Near-Term Solution for Efficient Merging of Aircraft on Uncoordinated Terminal RNAV Routes," Proceedings of $24^{\text {th }}$ Digital Avionics Systems Conference, Washington, D.C., 30 October - 5 November 2005.

21. MacWilliams, P.V., Smith, A.P., Becher, T.A., "RNP RNAV Arrival Route Coordination," Proceedings of $25^{\text {th }}$ Digital Avionics Systems Conference, Portland, OR, 15-19 October 2006.

22. Ren, L., and Clarke, J.-P., "A Separation Analysis Methodology for Designing Area Navigation Arrival Procedures," Journal of Guidance, Control, and Dynamics 30(5): 1319-1330 (September-October 2007).

23. Capozzi, B.J., Atkins, S.C., Choi, S., "Towards Optimal Routing and Scheduling of Metroplex Operations," Proceedings of9th AIAA Aviation Technology, Integration and Operations (ATIO) Conference, Paper 2009-7037, Hilton Head, SC, 21-23 September 2009.

24. Krozel, J., Weidner, T., and Hunter, G., “Terminal Area Guidance Incorporating Heavy Weather," AIAA Paper 97-3541, Aug., 1997.

25. Krozel, J., C. Lee, and J. S. B. Mitchell," "Turn-constrained route planning for avoiding hazardous weather," Air Traffic Control Quarterly, 14 (2), 2006, pp. 159-182.

26. Krozel, J., Penny, S., Prete, J., Mitchell, J.S.B., "Automated Route Generation for Avoiding Deterministic Weather in Transition Airspace," AIAA Journal of Guidance, Control, and Dynamics, Vol. 30 (1), 2007, pp. 144153..

27. Mitchell, J.S.B., Polishchuk, V., Krozel, J., "Airspace Throughput Analysis Considering Stochastic Weather," Proceeding of AIAA Guidance, Navigation, and Control (GNC) Conference, Keystone, CO. August 2006.

28. Steiner, M., Mueller, C., Davidson, G., and Krozel, J., "Integration of Probabilistic Weather Information with Air Traffic Management Decision Support Tools: A Conceptual Vision for the Future," 13th Conf. on Aviation, Range, and Aerospace Meteorology, American Meteorological Society, New Orleans, LA, 2008.

29. Rhoda, D. A., Pawlak, M. L., "An Assessment of Thunderstorm Penetrations and Deviations by Commercial Aircraft in the Terminal Area," Project Report NASA/A-2, MIT Lincoln Laboratory, Lexington, MA, 1999.

30. Michalek, D., Balakrishnan, H., "Dynamic Reconfiguration of Terminal Airspace During Convective Weather," Proceedings of the $49^{\text {th }}$ IEEE Conference on Decision and Control (submitted for publication), 15-17 December 2010.

31. Prevot, T., Callantine T, Kopardekar P, Smith N., Palmer E., and Battiste, V., "Trajectory-Oriented Operations with Limited Delegation: An Evolutionary Path to NAS Modernization," $4^{\text {th }}$ AIAA Aviation Technology, Integration and Operations (ATIO) Forum, AIAA-2004-6449, 2004.

32. Callantine, T.J., Lee, P. U., Mercer, J. S., Prevot, T., Palmer, E. A., "Air and Ground Simulation of Terminal-Area FMS Arrivals with Airborne Spacing and Merging," Proceedings of the 6th USA/Europe Air Traffic Management Research and Development Seminar, Baltimore, MD. 2005.

33. Lohr, G.W., Oseguera-Lohr, R.M., Abbott, T.S., Capron, W.R., “A Time-Based Airborne Inter-Arrival Spacing Tool: Flight Evaluation Results," Air Traffic Control Quarterly, Vol. 13(2) 2005.

34. Barmore, "Airborne Precision Spacing: A Trajectory-Based Approach to Improve Terminal Area Operations," $25^{\text {th }}$ Digital Avionics Systems Conference, Portland OR. 15-19 October 2006.

35. Erzberger, H., "Transforming the NAS: The Next Generation Air Traffic Control System," NASA/TP-2004212828 , Oct. 2004. 
36. Farley, T., Kupfer, M., and Erzberger, H., "Automated Conflict Resolution: A Simulation Evaluation Under High Demand Including Merging Arrivals," AIAA-2007-7736, 7th AIAA Aviation Technology, Integration, and Operations (ATIO) Conference, Belfast, Northern Ireland, 18-20 Sep. 2007.

37. Dear, R.. "The dynamic scheduling of aircraft in the near terminal area," FTL Report R76-9, Flight Transportation Laboratory, M.I.T, Cambridge, MA, August 1976.

38. Nedell, W., and Erzberger, H., "The Traffic Management Advisor," 1990 American Control Conference, San Diego, CA, May 1990.

39. Borchers, P. F. and Day, K., "Analysis of Divergences From Area Navigation Departure Routes at DFW Airport," Digial Avionics Systems Conference (DASC), Orlando, FL, 26-30 Oct. 2009.

40. C. Shiotsuki, G. C. Lin, and E. Hahn, "Analysis of operational impacts of continuous descent arrivals using Runway Simulator," The MITRE Corporation, F063-B07-058, 2007.

41. Erkelens, L.J.J., "Research into New Noise Abatement Procedures for the 21st Century," Proceedings of the AIAA Guidance, Navigation, and Control Conference, Denver, CO, AIAA-2000-4474, August 2000.

42. Shresta, S., Neskovic, D., and Williams, S., "Analysis of Continuous Descent Benefits and Impacts during Daytime Operations," Eighth USA/Europe Air Traffic Management Research and Development Seminar (ATM2009), Napa, CA, 2009.

43. Verhoeven, R.P.M, de Gelder, N., "Time-based navigation and ASAS interval managed CDA procedures," NLRTP-2009-477, September 2009.

44. Robinson, J. and Kamgarpour, M., "Benefits of Continuous Descent Operations in High-Density Terminal Airspace Under Scheduling Constraints," $10^{\text {th }}$ AIAA Aviation Technology, Integration, and Operations Conference (submitted for publication), Fort Worth, TX. Sept. 2010.

45. "Air Traffic Control Procedures and Phraseology," Federal Aviation Administration (FAA) Order JO 7110.65T, 11 February 2010.

46. Verma, S., Kozon, T., "Preliminary guidelines on controller's procedures for pairing aircraft for simultaneous approaches under different levels of automation," Proceedings of $3^{\text {rd }}$ International Conference on Applied Human Factors and Ergonomics (AHFE), Miami, FL, 17-20 July 2010.

47. Robinson III, J. E., and Isaacson, D. R., "A Concurrent Sequencing, and Deconfliction Algorithm for Terminal Area Air Traffic Control," AIAA Guidance, Navigation, and Control Conference, Denver, CO, Aug. 2000.

48. Barmore, B. E., Abbott, T.S., Capron, W. R. Baxley, B.T., "Simulation Results for Airborne Precision Spacing along Continuous Descent Arrivals," 8th AIAA Aviation Technology, Integration, and Operations (ATIO) Conference, Anchorage, AK, 14-19 September 2008.

49. "FAA's NextGen: FAA Response to Recommendations of the RTCA NextGen Mid-Term Implementation Task Force," Federal Aviation Administration, Washington, D.C. January 2010.

50. Tang, H., Robinson, J., Denery, D., "Tactical Conflict Detection in Terminal Airspace," $10^{\text {th }}$ AIAA Aviation Technology, Integration, and Operations Conference (submitted for publication), Fort Worth, TX. Sept. 2010.

51. Gong, C. and Sadovsky, A., "A Final Approach Trajectory Model for Current Operations," $10^{\text {th }}$ AIAA Aviation Technology, Integration, and Operations Conference (submitted for publication), Fort Worth, TX. Sept. 2010..

52. Denery, D. G., Tang, H. and Robinson, J.E., "Concept of Operation for Tactical Separation Assurance in SuperDensity Operations," NASA/TM-2010-216389, August 2010.

53. Zingale, C. M., Truitt, T. R., \& McAnulty, D. M., "Human-in-the-loop evaluation of an integrated arrival/departure air traffic control service for major metropolitan airspaces," DOT/FAA/TC-08/04, Atlantic City International Airport, NJ: Federal Aviation Administration William J. Hughes Technical Center, 2008.

54. Thippavong, J. and Mulfinger, D., "Design Considerations for a New Terminal Area Arrival Scheduler,". 10 AIAA Aviation Technology, Integration, and Operations Conference (submitted for publication), Fort Worth, TX. Sept. 2010.

55. Williams, D., "FMS VNAV Issues Affecting CDA RNAV Arrival Operations," Draft Report to JPDO NextGen Environmental Working Group, 25 September 2007.

56. Neuman, F., and Erzberger, H., "Analysis of Delay Reducing and Fuel Saving Sequencing and Spacing Algorithms for Arrival Traffic," NASA TM-103880, 1991.

57. Kupfer, M., Callantine, T.J., Mercer, J., Martin, L., and Palmer, E., "Controller-Managed Spacing - A Human-InThe-Loop Simulation of Terminal-Area Operations," AIAA Guidance, Navigation and Control Conference (submitted for publication), Toronto, Ontario, Canada, 2-5 August 2010.

58. Isaacson, D. R., and Robinson III, J. E., "A Knowledge-based Conflict Resolution Algorithm for Terminal Area Air Traffic Control Advisory Generation," AIAA Guidance, Navigation, and Control Conference, Montreal, Canada, Aug. 2001.

59. "Special Issue on Airborne Separation Assurance Systems (ASAS)," Air Traffic Control Quarterly, Vol. 13(2) 2005.

60. Erzberger, H., Heere, K., "Algorithm and Operational Concept for Resolving Short-Range Conflicts," Proc. IMechE Vol. 224 Part G: J. Aerospace Engineering, 2009.

61. Polishchuk, V., A., Mitchell, J.S.B., and Krozel, J., "Planning Routes with Wiggle Room in En Route WeatherImpacted Airspaces," AIAA Guidance, Navigation, and Control Conf., Chicago, IL, Aug., 2009. 
62. Krozel, J., Jakobovits, R., Mitchell, J.S.B., Kim, J., Zou, J., and Kicinger, R., "Resource Management to Address Weather Impacts in Super-Dense Terminal Airspaces: Software Prototype for National Flow Planning using Fixbased GDPs for Super Dense Terminal Operations," Technical Report, Metron Aviation, Inc., Dulles, VA, Aug. 29, 2009.

63. Verma, S., Lozito, S., and Trott, G., "Preliminary Guidelines on Flight Deck Procedures for Very Closely Spaced Parallel Approaches," International Council for the Aeronautical Sciences (ICAS) 2008 Congress, Anchorage, AK, 14-19 Sep. 2008.

64. "NextGen Integrated Work Plan," ver. 1.0, Joint Planning and Development Office (JPDO), Washington, D.C. 30 September 2008. 